\title{
Agradecimientos - Acknowledgements
}

\author{
Revista de la Academia Colombiana de Ciencias Exactas, Físicas y Naturales
}

Volumen 40, números 154, 155, 156 y 157.

El comité editorial de la revista de la Academia Colombiana de Ciencias Exactas, Físicas y Naturales expresa sincero agradecimiento a los revisores que han participado durante el año 2016, en la valoración crítica de los artículos presentados a la revista, por su valiosa contribución, esfuerzo y tiempo dedicado.

\author{
Agustín Cardina Molina \\ Alejandro Grajales \\ Alejandro Lopera \\ Alirio Palma \\ Álvaro Morales Aramburo \\ Andrea Polanco \\ Andrés Merchán \\ Ángela Camacho Beltrán \\ Arturo Acero \\ Augusto Rivera Umaña \\ Beatriz Martínez \\ Bernardo Uribe Jongbloed \\ Bibiana Moncada \\ Carlos Ávila \\ Carlos Flórez \\ Carlos Montoya \\ Carlos Parra Osorio \\ Carlos Rivera Rondón \\ Carmiña Gartner Vargas \\ Carmen Zúñiga de Cardozo \\ Catherine Graham \\ Cesar Vinasco \\ Constanza Ricaurte \\ David Arboleda \\ Diana Castaño \\ Diego Zarrate Charry \\ Diógenes Campos Romero \\ Edgar Cantillo \\ Edgardo Londoño-Cruz \\ Eduardo Brieva Bustillo \\ Edwin Munevar \\ Elisa Bonaccorso \\ Enrique Casanovas \\ Enrique Forero \\ Enrique Peña Salamanca \\ Erik Backelin \\ Fabio Cediel Melo \\ Fanor Mondragón \\ Fernando Alvarez-Valin \\ Fernando Guzmán \\ Gabriel Roldán
}

Gabriel Navas
Gaspar Monsalve Mejía
Gary Stiles Hurd
Gean Arteaga
Gerardo Aymard Corredor
Germán Chaparro Molano
Gianluca Lombardi
Gladys Reinoso
Héctor Ramírez Chávez
Héctor Sepúlveda Soto
Iván Correa
Ivonne Nieto
Jaime Forero
Jaime Vélez Upegui
Jairo Marín Cadavid
Javier Colmenares
Jereson Silva Valencia
Jesús Rangel
Johans Restrepo
Jorge Cossio
Jorge Henao-Mejía
Jorge Martínez Collantes
José García Varela
José Pabón
José Portilla Barbosa
José Rances Caicedo
Joshua Slattery
Juan Builes
Juan Camilo Dumar
Juan Cardona Gómez
Juan Cubillos Hinojosa
Juan de Sanctis
Juan Gallego Gómez
Juan Marín
Juan Pardo
Juan Restrepo
Juan Vélez Caicedo
Katherine Gross
Leonardo Castañeda Colorado
Lisa Campbell
Luis Barrera Avellaneda

Luis Caraballo

Luis Carlos Arboleda Aparicio

Luis Cuca

Luis Fernando García

Luz Veloza Castiblanco

Magnolia Longo

Malva Hernández

María Gabriela Fernández Pepi

Maribeb Castro-González

Martín Martín

Mikel Hurtado

Moisés Wasserman

Mónica Botero

Nathali López Cardona

Nelson Vera Villamizar

Nicolás Loaiza Díaz

Nicolau Saldanha

Oscar Arnache Olmos

Oscar Mesa Sánchez

Oscar Mosquera

Omar Triana

Paola Delgado

Patrick Druckenmiller

Pedro Dueñas Ramírez

Pedro Patarroyo

Rafael Baquero Parra

Rafael González Hernández

Ramón Henao

Richard Forrest

Roberto Martínez

Román Castañeda Sepúlveda

Rosario Medel

Rubén Vargas

Sandra Torijano

Santiago Vargas Domínguez

Sergio Restrepo Moreno

Sven Zea Sjorberg

Vladimir Garrido Ortega

Vladimir Kouznetsouv

Xavier Caicedo Ferrer

Ziv Arbeli 